\title{
Entropy and renormalized solutions for some nonlinear anisotropic elliptic equations with variable exponents and $L^{1}$-data
}

\author{
Mostafa El Moumni $^{1}$ and Deval Sidi Mohamed ${ }^{2}$
}

AвSTRACT. We prove in this paper some existence and unicity results of entropy and renormalized solutions for some nonlinear elliptic equations with general anisotropic diffusivities and variable exponents. The data are assumed to be merely integrable.

Mathematics Subject Classification (2020). 35J60, 46E35, 35D10.

Key words and phrases. Nonlinear elliptic equations, anisotropic diffusion, Variable exponents, Entropy solutions, Renormalized solution, $L^{1}$-data.

\section{Introduction}

This paper is motivated by the study whose model, including the stationary equation, is

$$
\left\{\begin{array}{l}
-\sum_{l=1}^{N} \frac{\partial}{\partial x_{l}}\left(\left|\frac{\partial u}{\partial x_{l}}\right|^{p_{l}(x)-2} \frac{\partial u}{\partial x_{l}}\right)+|u|^{s(x)-1} u=f \quad \text { in } \Omega, \\
u=0 \text { on } \partial \Omega
\end{array}\right.
$$

Received : August 31, 2020 - Accepted: January 1, 2021.

C) The Author(s) 2020. This article is published with open access by Sidi Mohamed Ben Abdallah University.

${ }^{1}$ Department of Mathematics, Faculty of Sciences El Jadida, University Chouaib Doukkali, P.O. Box 20, 24000 El Jadida, Morocco.

e-mail ${ }^{1}$ : mostafaelmoumni@gmail.com (Corresponding Author).

${ }^{2}$ Laboratory LAMA, Department of Mathematics, Faculty of Sciences Dhar El Mahraz, University Sidi Mohamed Ben Abdellah, P.O. Box 1796 Atlas Fez, Morocco.

e-mail ${ }^{2}$ : sidideval@gmail.com. 
where $N \geq 2$, the right-hand side $f \in L^{1}(\Omega)$ and $p_{l}: \bar{\Omega} \rightarrow(1,+\infty)$ is a continuous function for $l=1, \ldots, N$.

For $(1.1)$ we assume that the exponents functions $p_{1}(\cdot), \ldots, p_{N}(\cdot)$ and $s(\cdot)$ are restricted as follows:

$$
\left\{\begin{array}{l}
\bar{p}(\cdot)<N, \quad \frac{1}{\bar{p}(\cdot)}=\frac{1}{N} \sum_{l=1}^{N} \frac{1}{p_{l}(\cdot)} \\
p_{l}(\cdot)>1 \quad \text { and } \quad \frac{\bar{p}(\cdot)(N-1)}{N(\bar{p}(\cdot)-1)}<p_{l}(\cdot)<\frac{\bar{p}(\cdot)(N-1)}{N-\bar{p}(\cdot)}, \quad l=1, \ldots, N, \\
s(\cdot)>\max _{x \in \bar{\Omega}} p_{l}(x), \quad l=1, \ldots, N .
\end{array}\right.
$$

The study of the nonlinear partial differential equations in this type of spaces is strongly motivated by numerous phenomena of physics, namely the problems related to non-Newtonian fluids of strongly inhomogeneous behavior with a high ability of increasing their viscosity under a different stimulus, like the shear rate, magnetic or electric field. We use the framework of renormalized solutions. This notion was introduced by DiPerna and Lions [28] in their study of the Boltzmann equation, Boccardo et al [23] and Lions and Murat (see Lions book on the Navier-Stokes equations [37]). For the corresponding isotropic parabolic equations with $L^{1}$-data, existence and uniqueness of renormalized solutions is established in Blanchard and Murat [22], see also Rakotoson [38, 39] and Lions [37] for some time dependent problems motivated by the Navier-Stokes equations. Finally, let us mention that an equivalent notion of solutions, called entropy solutions, was introduced independently by Bénilan et al. [15], see also [11], several researches deals with the existence solutions of elliptic and parabolic problems under various assumptions and in different contexts (see $[42,32,43,6,8,19,1,7,5,2,16,9,17,20,3,10,4,18,30,31,29,25,21])$.

In the isotropic case with $p_{l}(\cdot)=p(\cdot)>2-\frac{1}{N}$ for $l=1, \ldots, N$, existence and regularity results are proved in $[14,40]$. The existence and regularity results for distributional solutions with $L^{1}$-data have been obtained in $[24,36]$ for a class of anisotropic elliptic and parabolic equations with constant variable components. For an anisotropic parabolic reaction-diffusionadvection system with a zero-flux boundary condition, similar results are established in [13]. Observe that (1.2) implies $\bar{p}(\cdot)>2-\frac{1}{N}$ and thus $\frac{N(\bar{p}(\cdot)-1)}{\bar{p}(N-1)} p_{l}(\cdot)>1$. This is in accordance with the isotropic case [14]. On the other hand, the condition $s(\cdot)>p_{l}(\cdot)$ in (1.2) is stronger than in [14]. This is a consequence of the anisotropic Sobolev inequality [41] (applied to our anisotropic equation)

The scope of the present paper is to solve the well-posdness (existence and uniquess) and regularity of entropy and renormalized solutions in an appropriate function space for nonlinear anisotropic elliptic equations with variable exponents. There are two difficulties associated with this strategy. In view of the assumption that $f$ is only integrable on $\Omega$, the first difficulty is to obtain suitable a priori estimates on $u_{\varepsilon}$ and the partial derivatives $\frac{\partial u_{\varepsilon}}{\partial x_{l}}, l=1, \ldots, N$, that are independent of $\varepsilon$. The second difficulty lies in passing to the limit in the nonlinear anisotropic vector field (with variable exponents) $A\left(x, \nabla u_{\varepsilon}\right)$ and the nonlinear term $\left|u_{\varepsilon}\right|^{s(\cdot)-1} u_{\varepsilon}$.

The paper is structured as follows: In Section 2 we recall some basic notations and a Sobolev inequality for anisotropic Sobolev spaces. Our main results are stated in Section 3, while the 
proofs are given in Section 4. In Section 5 we show the existing uniqueness renormalized solutions for elliptic equations. Finally, we prove equivalence between entropy and renormalized solutions to elliptic equations in Section 6.

\section{Sobolev spaces with variable exponents}

We recall in what follows some definitions and basic properties of the generalized LebesgueSobolev spaces $L^{p(\cdot)}(\Omega), W^{1, p(\cdot)}(\Omega)$ and $W_{0}^{1, p(\cdot)}(\Omega)$, where $\Omega$ is an open subset of $\mathbb{R}^{N}$. We refer to [34] and [33] for further properties of variable exponent Lebesgue-Sobolev spaces.

The continuous real-valued function $p: \bar{\Omega} \rightarrow[1,+\infty)$ satisfies the log-continuity if

$$
\forall x_{1}, x_{2} \in \bar{\Omega}, \quad\left|x_{1}-x_{2}\right|<1, \quad\left|p\left(x_{1}\right)-p\left(x_{2}\right)\right|<w\left(\left|x_{1}-x_{2}\right|\right),
$$

where $\limsup _{\alpha \rightarrow 0^{+}} w(\alpha) \ln \left(\frac{1}{\alpha}\right)<+\infty$. Let $p^{-}=\min _{x \in \bar{\Omega}} p(x)$ and $p^{+}=\max _{x \in \bar{\Omega}} p(x)$. We define the variable exponent Lebesgue space

$$
L^{p(\cdot)}(\Omega)=\left\{u: \Omega \rightarrow \mathbb{R} ; u \text { is measurable with } \int_{\Omega}|u(x)|^{p(x)} d x<\infty\right\} .
$$

We define a norm, the so-called Luxemburg norm, on this space by the formula

$$
\|u\|_{L^{p(\cdot)}(\Omega)}=\inf \left\{\mu>0 ; \int_{\Omega}\left|\frac{u(x)}{\mu}\right|^{p(x)} d x \leq 1\right\} .
$$

The following inequality will be used later

$$
\min \left\{\|u\|_{L^{p(\cdot)}(\Omega)}^{p^{-}},\|u\|_{L^{p(\cdot)}(\Omega)}^{p^{+}}\right\} \leq \int_{\Omega}|u(x)|^{p(x)} d x \leq \max \left\{\|u\|_{L^{p(\cdot)}(\Omega)}^{p^{-}},\|u\|_{L^{p(\cdot)}(\Omega)}^{p^{+}}\right\} .
$$

If $p^{-}>1$, then $L^{p(\cdot)}(\Omega)$ is reflexive and the dual space of $L^{p(\cdot)}(\Omega)$ can be identified with $L^{p^{\prime}(\cdot)}(\Omega)$, where $\frac{1}{p(\cdot)}+\frac{1}{p^{\prime}(\cdot)}=1$. For any $u \in L^{p(\cdot)}(\Omega)$ and $v \in L^{p^{\prime}(\cdot)}(\Omega)$ the Hölder type inequality

$$
\int_{\Omega}|u v| d x \leq\left(\frac{1}{p^{-}}+\frac{1}{p^{\prime-}}\right)\|u\|_{L^{p(\cdot)}(\Omega)}\|v\|_{L^{p^{\prime}(\cdot)}(\Omega)}
$$

holds true.

The inclusion between Lebesgue spaces also generalizes naturally: if $0<|\Omega|<\infty$ and $r_{1}$, $r_{2}$ are variable exponents so that $r_{1}(\cdot) \leq r_{2}(\cdot)$ almost everywhere in $\Omega$ then there exists the continuous embedding $L^{r_{2}(\cdot)}(\Omega) \hookrightarrow L^{r_{1}(\cdot)}(\Omega)$, whose norm does not exceed $|\Omega|+1$.

In this paper we will most exclusively work with Lebesgue and Lebesgue-Sobolev spaces with continuous variable exponents.

Now, we define also the variable Sobolev space

$$
W^{1, p(\cdot)}(\Omega)=\left\{u \in L^{p(\cdot)}(\Omega) ;|\nabla u| \in L^{p(\cdot)}(\Omega)\right\} .
$$


On $W^{1, p(\cdot)}(\Omega)$ we may consider one of the following equivalent norms

$$
\|u\|_{W^{1, p(\cdot)}(\Omega)}=\|u\|_{L^{p(\cdot)}(\Omega)}+\|\nabla u\|_{L^{p(\cdot)}(\Omega)} \cdot
$$

We define also $W_{0}^{1, p(\cdot)}(\Omega):={\overline{C_{c}^{\infty}(\Omega)}}^{W^{1, p(\cdot)}(\Omega)}$. Assuming $p^{-}>1$ the spaces $W^{1, p(\cdot)}(\Omega)$ and $W_{0}^{1, p(\cdot)}(\Omega)$ are separable and reflexive Banach spaces. The space $\left(W_{0}^{1, p(\cdot)}(\Omega)\right)^{\star}$ denotes the dual of $W_{0}^{1, p(\cdot)}(\Omega)$.

For $u \in W_{0}^{1, p(\cdot)}(\Omega)$ with $p \in C(\bar{\Omega})$ and $p^{-} \geq 1$, the Poincaré inequality holds (cf. [35])

$$
\|u\|_{L^{p(\cdot)}(\Omega)} \leq C\|\nabla u\|_{L^{p(\cdot)}(\Omega)},
$$

for some universal constant $C$ which depends on $\Omega$ and the function $p$.

For $p \in C\left(\bar{\Omega}, \mathbb{R}^{+}\right)$with $1<p^{-} \leq p^{+}<N$ the Sobolev embedding holds (see e.g. [33])

$$
W^{1, p(\cdot)}(\Omega) \subset L^{r(\cdot)}(\Omega),
$$

for any measurable function $r: \Omega \rightarrow[1,+\infty)$ such that

$$
\text { ess } \inf _{x \in \Omega}\left(\frac{N p(x)}{N-p(x)}-r(x)\right)>0 .
$$

Next, we recall the notion of anisotropic Sobolev spaces. Let $p_{1}, \ldots, p_{N}$ be $N$ real numbers with $p_{l} \geq 1, l=1, \ldots, N$. With a slight abuse of the notation, we introduce the anisotropic Sobolev space

and

$$
W^{1, p_{l}(\cdot)}(\Omega)=\left\{u \in L^{p_{l}(\cdot)}(\Omega): \frac{\partial u}{\partial x_{l}} \in L^{p_{l}(\cdot)}(\Omega)\right\},
$$

$$
W_{0}^{1, p_{l}(\cdot)}(\Omega)=\left\{u \in W_{0}^{1,1}(\Omega): \frac{\partial u}{\partial x_{l}} \in L^{p_{l}(\cdot)}(\Omega)\right\} .
$$

which are Banach spaces under the norm

$$
\|u\|=\|u\|_{L^{p_{l}(\cdot)}(\Omega)}+\left\|\frac{\partial u}{\partial x_{l}}\right\|_{L^{p_{l}(\cdot)}(\Omega)},
$$

for $l=1, \ldots, N$.

Let us recall the anisotropic Sobolev imbedding theorems (with constant variable exponents) due to Troisi [41].

Theorem 2.1. Suppose $g \in \bigcap_{l=1}^{N} W_{0}^{1, p_{l}}(\Omega)$, and let

$$
\begin{cases}q=\bar{p}^{\star}, & \text { if } \bar{p}^{\star}<N, \\ q \in[1, \infty), & \text { if } \bar{p}^{\star} \geq N .\end{cases}
$$

Then there exists a constant $C$, depending on $N, p_{1}, \ldots, p_{N}$ if $\bar{p}<N$ and also on $q$ and $|\Omega|$ if $\bar{p} \geq N$, such that

$$
\|g\|_{L^{q}(\Omega)} \leq C \prod_{l=1}^{N}\left\|\frac{\partial g}{\partial x_{l}}\right\|_{L^{p_{l}(\Omega)}}^{1 / N} .
$$


Theorem 2.2. Let $Q$ be a cube of $\mathbb{R}^{N}$ with faces parallel to the coordinate planes. Suppose $u \in$ $N$ $\bigcap_{l=1} W^{1, p_{l}}(Q)$, and set

$$
\frac{1}{\bar{p}}=\frac{1}{N} \sum_{l=1}^{N} \frac{1}{p_{l}}, \quad r= \begin{cases}\bar{p}^{\star}:=\frac{N \bar{p}}{N-\bar{p}}, & \text { if } \bar{p}^{\star}<N, \\ \text { any number from }[1, \infty), & \text { if } \bar{p}^{\star} \geq N .\end{cases}
$$

Then there exists a constant $C$, depending on $N, p_{1}, \ldots, p_{N}$ if $\bar{p}<N$ and also on $r$ and meas $(Q)$ if $\bar{p} \geq N$, such that

$$
\|u\|_{L^{r}(Q)} \leq C \prod_{l=1}^{N}\left[\left\|\frac{\partial u}{\partial x_{l}}\right\|_{L^{p_{l}(Q)}}+\|u\|_{L^{p_{l}(Q)}}\right]^{\frac{1}{N}} .
$$

Theorems 2.1 and 2.2 will be used to prove the "interpolation" lemma below, which is a technical result we will use later to obtain a priori estimates.

\section{Statements of results}

Instead of (1.1) we will consider more general nonlinear anisotropic elliptic equations with variable exponents of the form

$$
-\operatorname{div} A(x, \nabla u)+g(x, u)=f(x) \quad \text { in } \Omega .
$$

The vector field $A: \Omega \times \mathbb{R}^{N} \rightarrow \mathbb{R}^{N}$ has components $a_{l}: \Omega \times \mathbb{R}^{N} \rightarrow \mathbb{R}, l=1, \ldots, N$, and we assume that there exist two constants $C_{A}$ and $C_{A}^{\prime}$ such that for all $\xi_{1}, \xi_{2} \in \mathbb{R}^{N}$ and for a.e. $x$

$$
\begin{aligned}
& A(x, \xi) \cdot \xi \geq C_{A} \sum_{l=1}^{N}|\xi|^{p_{l}(x)}, \\
& \left|a_{l}(x, \xi)\right| \leq C_{A}^{\prime}\left(1+\sum_{\ell=1}^{N}\left|\xi_{l}\right|^{p_{l}(x)-1}\right), \quad l=1, \ldots, N, \\
& {\left[A\left(x, \xi_{1}\right)-A\left(x, \xi_{2}\right)\right]\left[\xi_{1}-\xi_{2}\right]>0, \quad \xi_{1} \neq \xi_{2} .}
\end{aligned}
$$

The nonlinear function $g: \Omega \times \mathbb{R} \rightarrow \mathbb{R}$ is assumed to be measurable in $x \in \Omega$ for all $\sigma \in \mathbb{R}$ and continuous in $\sigma \in \mathbb{R}$ for a.e. $x \in \Omega$. Furthermore,

$$
\begin{aligned}
& g(x, \sigma) \sigma \geq 0, \quad \text { for all } \sigma \in \mathbb{R} \text { and a.e. } x \in \Omega, \\
& \sup \{|g(x, \sigma)|:|\sigma| \leq \tau\} \in L^{1}(\Omega), \quad \forall \tau \in \mathbb{R} .
\end{aligned}
$$

Finally, there should exist $s>p_{l}, l=1, \ldots, N$, such that

$$
g(x, \sigma) \operatorname{sign}(\sigma) \geq|\sigma|^{s(x)}, \quad \text { for all } \sigma \in \mathbb{R} \text { and a.e. } x \in \Omega \text {. }
$$

For any given $\gamma>0$, we define the truncation function $T_{\gamma}: \mathbb{R} \rightarrow \mathbb{R}$ by

$$
T_{\gamma}(z)= \begin{cases}-\gamma, & \text { if } z \leq-\gamma \\ z, & \text { if }|z|<\gamma \\ \gamma, & \text { if } z \geq \gamma\end{cases}
$$


Moreover, we will need the following associated function (renormalization)

$$
\phi_{\gamma}(r)=T_{\gamma+1}(r)-T_{\gamma}(r),
$$

for any $\gamma>0$. Notice that $T_{\gamma}$ and $\phi_{\gamma}$ are Lipschitz functions satisfying $\left|T_{\gamma}(r)\right| \leq \gamma$ and $\left|\phi_{\gamma}(r)\right| \leq 1$.

Pick any positive $C^{\infty}(\mathbb{R})$ function $s(\cdot)$ such that $s(z)=1$ if $|z| \leq 1, s(z)=0$ if $|z| \geq 2$, and $0 \leq s(z) \leq 1$ for all $z \in \mathbb{R}$. For any $n \geq 2$, define the function $S_{n}(r)$ by $S_{n}(r)=\int_{0}^{r} s_{n}(z) d z$, where

$$
s_{n}(z)= \begin{cases}1, & \text { if }|z| \leq n-1 \\ s(z-(n-1) \operatorname{sign}(z)), & \text { if }|z| \geq n-1\end{cases}
$$

where $\operatorname{sign}(z)$ denotes the sign of $z$. For each integer $n \geq 2$, the function $S_{n}$ satisfies

$$
\begin{cases}S_{n}(r)=S_{n}\left(T_{n+1}(r)\right), & \left\|S_{n}^{\prime}\right\|_{L^{\infty}(\mathbb{R})} \leq\|s\|_{L^{\infty}(\mathbb{R}),} \\ \operatorname{supp} S_{n}^{\prime} \subset[-(n+1), n+1], & \operatorname{supp} S_{n}^{\prime \prime} \subset[-(n+1),-n] \cup[n, n+1] .\end{cases}
$$

We shall use the following definitions of entropy solutions and renormalized solutions for problem (3.1) in the following sense:

Definition 3.1. An entropy solution of (3.1) is a function $u: \mathbb{R}^{N} \rightarrow \mathbb{R}$ such

$$
u \in W_{0}^{1,1}(\Omega) \cap L^{s(\cdot)}(\Omega), \quad A(x, \nabla u) \in\left(L^{1}(\Omega)\right)^{N},
$$

and $\forall \varphi \in C_{c}^{1}(\Omega)$

$$
\int_{\Omega} A(x, \nabla u) \cdot \nabla T_{\gamma}(u-\varphi) d x+\int_{\Omega} g(x, u) T_{\gamma}(u-\varphi) d x=\int_{\Omega} f T_{\gamma}(u-\varphi) d x .
$$

Definition 3.2. A renormalized solution of (3.1) is a function $u \in L^{1}(\Omega)$, satisfying the following conditions:

and

$$
T_{\gamma}(u) \in W_{0}^{1, p(\cdot)}(\Omega) \text { for any } \gamma>0
$$

$$
\lim _{\gamma \rightarrow \infty} \sum_{l=1}^{N} \int_{\{\gamma \leq|u| \leq \gamma+1\}}\left|\frac{\partial u}{\partial x_{l}}\right|^{p_{l}(x)} d x=0 .
$$

For any renormalization $S \in C^{\infty}(\mathbb{R})$ such that supp $S^{\prime} \subset[-M, M]$ for some $M>0$,

$$
-\sum_{l=1}^{N} \frac{\partial}{\partial x_{l}}\left(S^{\prime}(u)\left|\frac{\partial u}{\partial x_{l}}\right|^{p_{l}(x)-2} \frac{\partial u}{\partial x_{l}}\right)+\sum_{l=1}^{N} S^{\prime \prime}(u)\left|\frac{\partial u}{\partial x_{l}}\right|^{p_{l}(x)}+g(x, u) S^{\prime}(u)=f S^{\prime}(u) \text { in } \mathcal{D}^{\prime}(\Omega) \text {. }
$$

Our main results are collected in the following theorem:

Theorem 3.1. Assume (3.2)-(3.7) hold and the corresponding exponents functions $p_{1}(\cdot), \ldots, p_{N}(\cdot)$ and $s(\cdot)$ are restricted as in (1.2). Let $f \in L^{1}(\Omega)$. Then (3.1) has at least one entropy and renormalized solution $u$.

Remark 3.1. The notion of entropy and renormalized solution for problem (3.1) are is equivalent. 


\section{Proof of Theorem 3.1}

Let $\left(f_{\varepsilon}\right)_{0<\varepsilon<1} \subset C_{c}^{\infty}(\Omega)$ be a sequence of smooth approximations of $f$ such that

$$
\left\{\begin{array}{l}
\left|f_{\varepsilon}\right| \leq \frac{1}{\varepsilon} \quad \text { and } \quad\left|f_{\varepsilon}\right| \leq|f| \\
f_{\varepsilon} \rightarrow f \text { in } L^{1}(\Omega) \quad \text { as } \varepsilon \rightarrow 0 .
\end{array}\right.
$$

Then the result in [14], provide us with the existence of a sequence of functions

$$
\left(u_{\varepsilon}\right)_{0<\varepsilon \leq 1} \subset \bigcap_{l=1}^{N} W_{0}^{1, p_{l}(\cdot)}(\Omega) \cap L^{s(\cdot)}(\Omega),
$$

each of them satisfying the weak formulation

$$
\int_{\Omega} A\left(x, \nabla u_{\varepsilon}\right) \cdot \nabla \varphi d x+\int_{\Omega} g\left(x, u_{\varepsilon}\right) \varphi=\int_{\Omega} f_{\varepsilon} \varphi d x,
$$

for all $\varphi \in \bigcap_{l=1}^{N} W_{0}^{1, p_{l}(\cdot)}(\Omega) \cap L^{\infty}(\Omega)$. We prove Theorem 3.1 in three main steps. We prove first $\varepsilon$-uniform a priori estimates for $u_{\varepsilon}$, so we obtain a.e. convergence of $u_{\varepsilon}$. In the second step, we prove strong $L^{1}$ convergence of the nonlinear term $g$ in (4.2). Finally, we complete the proof of Theorem 3.1 by passing to the limit in (4.1) as $\varepsilon \rightarrow 0$.

In the remaining part of this paper, we use $C, C_{1}, C_{2}$, etc. to denote constants that are independent of $\varepsilon$.

\subsection{A priori estimates.}

Proposition 4.1. Assume (3.2)-(3.7) hold, and that the exponents $p_{1}(\cdot), \ldots, p_{N}(\cdot)$ and $s(\cdot)$ are restricted as in (1.2). Then, there exist a constant $C$, not depending on $\varepsilon$, such that

$$
\begin{gathered}
\sum_{l=1}^{N} \int_{\left\{\left|u_{\varepsilon}\right| \leq \gamma\right\}}\left|\frac{\partial u_{\varepsilon}}{\partial x_{l}}\right|^{p_{l}(x)} d x \leq C \gamma, \quad \forall \gamma>0 \\
\left\|g\left(x, u_{\varepsilon}\right)\right\|_{L^{1}(\Omega)} \leq C
\end{gathered}
$$

and

$$
\sum_{l=1}^{N} \int_{\Omega} \frac{\left|\frac{\partial u_{\varepsilon}}{\partial x_{l}}\right|^{p_{l}(x)}}{\left(1+\left|u_{\varepsilon}\right|\right)^{\gamma}} d x \leq C, \quad \forall \gamma>1 .
$$

Proof. Inserting $\varphi=T_{\gamma}\left(u_{\varepsilon}\right)$ into (4.2) gives

$$
\int_{\Omega} A\left(x, \nabla u_{\varepsilon}\right) \cdot \nabla T_{\gamma}\left(u_{\varepsilon}\right) d x+\int_{\Omega} g\left(x, u_{\varepsilon}\right) T_{\gamma}\left(u_{\varepsilon}\right) d x=\int_{\Omega} f_{\varepsilon} T_{\gamma}\left(u_{\varepsilon}\right) d x .
$$

From the definition of $T_{\gamma}$ and $g$ the second integral is nonnegative, and since $\left|f_{\varepsilon}\right| \leq|f|$, we get (4.3). 
To prove (4.5), we introduce for $\gamma>1$ the test function

$$
\varphi_{\gamma}(\sigma)= \begin{cases}(\gamma-1) \int_{0}^{\sigma} \frac{1}{(1+t)^{\gamma}} d t=1-\frac{1}{(1+\sigma)^{\gamma-1}}, & \sigma \geq 0 \\ -\varphi_{\gamma}(-\sigma), & \sigma<0\end{cases}
$$

Substituting $\varphi=\varphi_{\gamma}\left(u_{\varepsilon}\right)$ in (4.2) gives

$$
\int_{\Omega} A\left(x, \nabla u_{\varepsilon}\right) \cdot \nabla u_{\varepsilon} \varphi_{\gamma}^{\prime}\left(u_{\varepsilon}\right) d x+\int_{\Omega} g\left(x, u_{\varepsilon}\right) \varphi_{\gamma}\left(u_{\varepsilon}\right) d x=\int_{\Omega} f_{\varepsilon} \varphi_{\gamma}\left(u_{\varepsilon}\right) d x .
$$

Using the structure condition (3.2) in (4.7), we get

$$
C_{A} \int_{\Omega} \sum_{l=1}^{N} \frac{\left|\frac{\partial u_{\varepsilon}}{\partial x_{l}}\right|^{p_{l}(x)}}{\left(1+\left|u_{\varepsilon}\right|\right)^{\gamma}} d x \leq\|f\|_{L^{1}(\Omega)}
$$

This gives (4.5).

Next, we take $\varphi=\frac{1}{\gamma} T_{\gamma}\left(u_{\varepsilon}\right)$ in (4.2). After letting $\gamma \rightarrow \infty$ we find

$$
\int_{\Omega}\left|g\left(x, u_{\varepsilon}\right)\right| d x \leq\|f\|_{L^{1}(\Omega)}
$$

which yields (4.4).

To continue we need the following lemma.

Lemma 4.1. Let $p_{l}(\cdot)$ be a continuous function on $\bar{\Omega}$ for $l=1, \ldots, N$, and $u_{\varepsilon}$ a function in $\bigcap_{l=1}^{N} W_{0}^{1, p_{l}(\cdot)}(\Omega)$. Suppose that the exponents $p_{1}(\cdot), \ldots, p_{N}(\cdot)$ and $s(\cdot)$ are restricted as in (1.2), and that there exists a constant $c$ such that

$$
\left\|u_{\varepsilon}\right\|_{L^{s(\cdot)}(\Omega)} \leq \mathcal{C}
$$

and

$$
\sum_{l=1}^{N} \int_{\Omega} \frac{\left|\frac{\partial u_{\varepsilon}}{\partial x_{l}}\right|^{p_{l}(x)}}{\left(1+\left|u_{\varepsilon}\right|\right)^{\gamma}} d x \leq c
$$

Then for all continuous functions $q_{l}(\cdot)$ on $\bar{\Omega}$ such that

$$
1 \leq q_{l}(x)<\frac{N(\bar{p}(x)-1)}{\bar{p}(x)(N-1)} p_{l}(x), \quad l=1, \ldots, N,
$$

there exists a constant $C$, depending on $\Omega, N, p_{1}(\cdot), \ldots, p_{N}(\cdot), q_{1}(\cdot), \ldots, q_{N}(\cdot)$, and $c$, such that

$$
\left\|\frac{\partial u_{\varepsilon}}{\partial x_{l}}\right\|_{L^{q_{l}(\cdot)}(\Omega)} \leq C, \quad l=1, \ldots, N,
$$

and

$$
\left\|u_{\varepsilon}\right\|_{L^{\bar{q}(\cdot)}(\Omega)} \leq C, \quad \frac{1}{\bar{q}(\cdot)}=\frac{1}{N} \sum_{l=1}^{N} \frac{1}{q_{l}(\cdot)} .
$$


Proof. In a first step let $q_{l}^{+}$be a constant satisfying (recall that $q_{l}^{+}=\max _{x \in \bar{\Omega}} q_{l}(x)$ and $p_{l}^{-}=$ $\left.\min _{x \in \bar{\Omega}} p_{l}(x)\right)$

$$
1 \leq q_{l}^{+}<\frac{N\left(\bar{p}^{-}-1\right)}{\bar{p}^{-}(N-1)} p_{l}^{-} .
$$

Observe that (4.15) implies $q_{l}^{+}<p_{l}^{-}$. Now let $q_{l}^{+}=\kappa p_{l}^{-}, l=1, \ldots, N$, for any $\kappa \in\left(0, \frac{N\left(\bar{p}^{-}-1\right)}{\bar{p}^{-}(N-1)}\right)$ where $\frac{1}{\bar{p}^{-}}=\frac{1}{N} \sum_{l=1}^{N} \frac{1}{p_{l}^{-}}$. Let $\gamma=\frac{1-\kappa}{\kappa} \overline{q^{+}}{ }^{\star}$, so that $\gamma \frac{q_{l}^{+}}{p_{l}^{-}-q_{l}^{+}}=\overline{q^{+}}{ }^{\star}$, where

$$
\overline{q^{+}}=\frac{N \overline{q^{+}}}{N-\overline{q^{+}}} \text {and } \frac{1}{\overline{q^{+}}}=\frac{1}{N} \sum_{l=1}^{N} \frac{1}{q_{l}^{+}} .
$$

Recalling $\frac{p_{l}^{-}-q_{l}^{+}}{q_{l}^{+}} \bar{q}^{\star}>1$, we see that $\gamma>1$. Using (4.11) and Hölder inequality, we get

$$
\begin{aligned}
\int_{\Omega}\left|\frac{\partial u_{\varepsilon}}{\partial x_{l}}\right|^{q_{l}^{+}} d x & \leq\left(\int_{\Omega} \frac{\left|\frac{\partial u_{\varepsilon}}{\partial x_{l}}\right|^{p_{l}^{-}}}{\left(1+\left|u_{\varepsilon}\right|\right)^{\gamma}} d x\right)^{\frac{q_{l}^{+}}{p_{l}^{-}}}\left(\int_{\Omega}\left(1+\left|u_{\varepsilon}\right|\right)^{\gamma \frac{q_{l}^{+}}{p_{l}^{-}-q_{l}^{+}}} d x\right)^{\frac{p_{l}^{-}-q_{l}^{+}}{p_{l}^{-}}} \\
& \leq C_{1}\left(\int_{\Omega}\left(1+\left|u_{\varepsilon}\right|\right)^{\gamma \frac{q_{l}^{+}}{p_{l}^{-}-q_{l}^{+}}} d x\right)^{\frac{p_{l}^{-}-q_{l}^{+}}{p_{l}^{-}}} \\
& \leq C_{2}\left(\int_{\Omega}\left|u_{\varepsilon}\right|^{q^{\star}} d x\right)^{\frac{p_{l}^{-}-q_{l}^{+}}{p_{l}^{-}}}+C_{3} .
\end{aligned}
$$

Using (4.16) and the anisotropic Sobolev inequality (2.6), we deduce

$$
\begin{aligned}
\left(\int_{\Omega}\left|u_{\varepsilon}\right|^{\overline{q^{+}}} d x\right)^{\frac{1}{q^{+}}} & \leq c_{3}+c_{4} \prod_{l=1}^{N}\left(\int_{\Omega}\left|u_{\varepsilon}\right|^{\overline{q^{+}}} d x\right)^{\frac{p_{l}^{-}-q_{l}^{+}}{q_{l}^{+} p_{l}^{-} N}} \\
& =c_{3}+c_{4}\left(\int_{\Omega}\left|u_{\varepsilon}\right|^{\overline{q^{+}}} d x\right)^{\sum_{l=1}^{N} \frac{p_{l}^{-}-q_{l}^{+}}{q_{l}^{+} p_{l}^{-} N}} \\
& =c_{3}+c_{4}\left(\int_{\Omega}\left|u_{\varepsilon}\right|^{\overline{q^{\star}}} d x\right)^{\frac{1}{\bar{q}^{+}}-\frac{1}{\bar{p}^{-}}} .
\end{aligned}
$$

for some constants $c_{3}, c_{4}>0$. In other words,

$$
\left\|u_{\varepsilon}\right\|_{L^{\bar{q}^{\star}}(\Omega)} \leq c_{5}+c_{6}\left\|u_{\varepsilon}\right\|_{L^{\bar{q}^{\star}}(\Omega)}^{a}, \quad a:=\frac{\bar{p}^{-}-\bar{q}^{+}}{\bar{q}^{+} \bar{p}^{-}} \overline{\bar{q}^{+}} .
$$


for some constants $c_{5}, c_{6}>0$. One checks easily that the assumption $\bar{p}<N$ implies $a<1$, and we can therefore conclude that for all $1 \leq q_{l}^{+}<\frac{N\left(\bar{p}^{-}-1\right)}{\bar{p}^{+}(N-1)} p_{l}^{-}$,

$$
\left\|\frac{\partial u_{\varepsilon}}{\partial x_{l}}\right\|_{L^{q_{l}^{+}(\Omega)}} \leq C, \quad l=1, \ldots, N,
$$

and

$$
\left\|u_{\varepsilon}\right\|_{L^{q^{+}(\cdot)}(\Omega)} \leq C, \quad \frac{1}{\overline{q^{+}(\cdot)}}=\frac{1}{N} \sum_{l=1}^{N} \frac{1}{q_{l}^{+}(\cdot)} .
$$

for some constant $C>0$.

Now let us consider a continuous variable exponent $q_{l}$ on $\bar{\Omega}$ satisfying the pointwise estimate (4.12) for $l=1, \ldots, N$. By the continuity of $p_{l}(\cdot)$ and $q_{l}(\cdot)$ on $\bar{\Omega}$ there exists a constant $\delta>0$ such that

$$
\max _{y \in \bar{Q}(x, \delta) \cap \Omega} q(y)<\min _{y \in \bar{Q}(x, \delta) \cap \Omega} \frac{N(\bar{p}(y)-1)}{\bar{p}(y)(N-1)} p_{l}(y), \quad l=1, \ldots, N .
$$

Herein, $Q(x, \delta)$ is a cube with center $x$ and diameter $\delta$. Observe that $\bar{\Omega}$ is compact and therefore we can cover it with a finite number of cubes $\left(Q_{i}\right)_{i=1, \ldots, k}$ with edges parallel to the coordinate axes, such that there exists a constant $\alpha>0$ such that

$$
\alpha<\operatorname{meas}\left(Q_{i} \cap \Omega\right)<\delta \text { for all } i=1, \ldots, k .
$$

We denote by $q_{i, l}^{-}$and $q_{i, l}^{+}$(respectively $p_{i, l}^{-}$and $p_{i, l}^{+}$) the local minimum and the local maximum of $q_{l}$ on $\overline{Q_{i} \cap \Omega}$ ( respectively the local minimum and the local maximum of $p_{l}$ on $\overline{Q_{i} \cap \Omega}$ ) for $l=1, \ldots, N$.

Using now the same arguments as before locally, we see that the inequality (4.16) holds on $Q_{i} \cap \Omega$, respectively. The anisotropic Sobolev inequality (2.7) gives

$$
\left(\int_{Q_{i} \cap \Omega}\left|u_{\varepsilon}\right|^{\overline{q_{i}^{+}}} d x\right)^{\frac{1}{q_{i}^{+\star}}} \leq c_{7} \prod_{l=1}^{N}\left[\left(\int_{Q_{i} \cap \Omega}\left|\frac{\partial u_{\varepsilon}}{\partial x_{l}}\right|^{q_{i, l}^{+}} d x\right)^{\frac{1}{q_{i, l}^{+}}}+\left(\int_{Q_{i} \cap \Omega}\left|u_{\varepsilon}\right|^{q_{i, l}^{+}} d x\right)^{\frac{1}{q_{i, l}^{+}}}\right]^{\frac{1}{N}},
$$

for some constant $c_{7}>0$. Since $q_{l}(\cdot)<s(\cdot)$ for $l=1, \ldots, N$, it follows from (4.20) and (4.10) that

$$
\left(\int_{Q_{i} \cap \Omega}\left|u_{\varepsilon}\right|^{\overline{q_{i}^{+}}} d x\right)^{\frac{1}{q_{i}^{+\star}}} \leq c_{7} \prod_{l=1}^{N}\left(\int_{Q_{i} \cap \Omega}\left|\frac{\partial u_{\varepsilon}}{\partial x_{l}}\right|^{q_{i, l}^{+}} d x\right)^{\frac{1}{q_{i, l}^{+} N}}+c_{8} .
$$

for some constant $c_{8}>0$.

Keeping in mind (4.18), we deduce from (4.17) (with $\bar{q}=\bar{q}_{i}, p_{l}=p_{i, l}$ and $q_{l}=q_{i, l}$ for $i=1, \ldots, k$ and $l=1, \ldots, N)$ and $(4.21)$

$$
\left\|u_{\varepsilon}\right\|_{L^{q_{i}^{+}}\left(Q_{i} \cap \Omega\right)} \leq c_{9}+c_{10}\left\|u_{\varepsilon}\right\|_{L^{q_{i}^{+}}\left(Q_{i} \cap \Omega\right)}^{a}, \quad a:=\frac{\overline{p_{i, l}}-{\overline{q_{i, l}}}^{+}}{{\overline{q_{i, l}}}^{+} \overline{p_{i, l}}-{ }_{i}^{+}},
$$


for some constants $c_{9}, c_{10}>0$. Obviously, this implies that, for some constant $C$, depending on $p_{i, l}, q_{i, l}$ and $\Omega$,

$$
\left\|\frac{\partial u_{\varepsilon}}{\partial x_{l}}\right\|_{L^{q_{i, l}}\left(Q_{i} \cap \Omega\right)} \leq C, \quad l=1, \ldots, N, \quad i=1, \ldots, k,
$$

and

$$
\left\|u_{\varepsilon}\right\|_{L_{L_{i}^{q^{+}}}\left(Q_{i} \cap \Omega\right)} \leq C, \quad \frac{1}{\overline{q_{i}^{+}}}=\frac{1}{N} \sum_{l=1}^{N} \frac{1}{q_{i, l}^{+}}, \quad i=1, \ldots, k .
$$

Finally, since $\overline{q_{i}^{+}}=\frac{N \overline{q_{i}^{+}}}{N-\overline{q_{i}^{+}}} \geq \bar{q}^{\star}(x) \geq \bar{q}(x)$ and $q_{i, l}^{+} \geq q_{l}(x)$ for all $x \in Q_{i} \cap \Omega$ and for all $i=1, \ldots, k$, we conclude from (4.22) and (4.23) that for $l=1, \ldots, N$,

$$
\left\|u_{\mathcal{E}}\right\|_{L^{\bar{q}^{\star}(\cdot)}(\Omega)}+\left\|\frac{\partial u_{\varepsilon}}{\partial x_{l}}\right\|_{L^{q_{l}(\cdot)}(\Omega)} \leq c_{11}
$$

for some constant $c_{11}$ depending on $p_{l}(\cdot), q_{l}(\cdot)$ and $\Omega$. This concludes the proof of the lemma for (4.11).

Observe that the consequence of Proposition 4.1 and Lemma 4.1: there exists a constant $C$, depending on $\Omega, N, p_{1}(\cdot), \ldots, p_{N}(\cdot), q_{1}(\cdot), \ldots, q_{N}(\cdot),\|f\|_{L^{1}(\Omega)}$ but not $\varepsilon$, such that

$$
\left\|\frac{\partial u_{\varepsilon}}{\partial x_{l}}\right\|_{L^{q_{l}(\cdot)}(\Omega)} \leq C, \quad l=1, \ldots, N
$$

and

$$
\left\|u_{\varepsilon}\right\|_{L^{\bar{q}(\cdot)}(\Omega)} \leq C, \quad \frac{1}{\bar{q}(\cdot)}:=\frac{1}{N} \sum_{l=1}^{N} \frac{1}{q_{l}(\cdot)}
$$

for every continuous function $q_{l}(\cdot)$ for $l=1, \ldots, N$, such that $1 \leq q_{l}(\cdot)<\frac{N(\bar{p}(\cdot)-1)}{\bar{p}(\cdot)(N-1)} p_{l}(\cdot)$.

4.2. Strong convergence. In view of Proposition $4.1, u_{\varepsilon}$ is uniformly (in $\varepsilon$ ) bounded in $W^{1, q_{0}}(\Omega)$, where

$$
q_{0}^{-}:=\min _{x \in \bar{\Omega}} q_{l}(\cdot) \text { for } l=1, \ldots, N,
$$

and $q_{1}(\cdot), \ldots, q_{N}(\cdot)$ are restricted as in Lemma 4.1. Without loss of generality, we can therefore assume that

$$
\left\{\begin{array}{l}
u_{\varepsilon} \rightarrow u \text { strongly in } L^{q_{0}^{-}}(\Omega) \text { and a.e. in } \Omega, \\
g\left(x, u_{\varepsilon}\right) \rightarrow g(x, u) \text { a.e. in } \Omega, \\
T_{\gamma}\left(u_{\varepsilon}\right) \rightarrow T_{\gamma}(u) \text { weakly in } \bigcap_{l=1}^{N} W_{0}^{1, p_{l}(\cdot)}(\Omega) .
\end{array}\right.
$$

Next, we need to prove the strong convergence in $L^{1}(\Omega)$ of the sequence $\left(g\left(x, u_{\varepsilon}\right)\right)_{0<\varepsilon \leq 1}$ to $g(x, u)$. 
Proposition 4.2. Assume (3.2)-(3.7) hold, and that the corresponding exponents $p_{1}(\cdot), \ldots, p_{N}(\cdot)$ and $s(\cdot)$ are restricted as in (1.2). Then the sequence $\left(g\left(x, u_{\varepsilon}\right)\right)_{0<\varepsilon \leq 1}$ converges to $g(x, u)$ a.e. in $\Omega$ and strongly in $L^{1}(\Omega)$.

The prove of Proposition 4.2 is similar to this in [12], so we omit the proof.

Lemma 4.2. The sequence $\left(u_{\varepsilon}\right)_{0 \leq \varepsilon<1}$ satisfies the following energy estimate

$$
\lim _{n \rightarrow \infty} \limsup _{\varepsilon \rightarrow 0} \int_{\left\{n \leq\left|u_{\varepsilon}\right| \leq n+1\right\}} \sum_{l=1}^{N}\left|\frac{\partial u_{\varepsilon}}{\partial x_{l}}\right|^{p_{l}(x)} d x=0 .
$$

Proof. Let $\phi_{n}$ be as defined in Subsection 2. Substituting $\varphi=\phi_{n}\left(u_{\varepsilon}\right)$ into (4.2) yields

$$
\int_{\Omega} A\left(x, \nabla u_{\varepsilon}\right) \cdot \nabla \phi_{n}\left(u_{\varepsilon}\right) d x+\int_{\Omega} g\left(x, u_{\varepsilon}\right) \phi_{n}\left(u_{\varepsilon}\right) d x=\int_{\Omega} f_{\varepsilon} \phi_{n}\left(u_{\varepsilon}\right) d x .
$$

Since $\frac{\partial u_{\varepsilon}}{\partial x_{l}} \phi_{n}\left(u_{\varepsilon}\right)=\mathbf{1}_{\left\{n \leq\left|u_{\varepsilon}\right| \leq n+1\right\}} \frac{\partial u_{\varepsilon}}{\partial x_{l}}$ a.e. in $\Omega$, we deduce from this that

$$
\limsup _{\varepsilon \rightarrow 0} \int_{\left\{n \leq\left|u_{\varepsilon}\right| \leq n+1\right\}} \sum_{l=1}^{N}\left|\frac{\partial u_{\varepsilon}}{\partial x_{l}}\right|^{p_{l}(x)} d x \leq \frac{1}{C_{A}} \int_{\Omega}|f| \phi_{n}(u) d x .
$$

Since $u \in L^{1}(\Omega)$, we obtain the a.e. convergence of $\phi_{n}(u)$ to 0 as $n \rightarrow \infty$. Consequently as $\left|\phi_{n}\right| \leq 1$, by Lebesgue Theorem we deduce $\int_{\Omega}|f| \phi_{n}(u) d x \rightarrow 0$ as $n \rightarrow \infty$. Passing to the limit $n \rightarrow \infty$ in (4.28) yields the desired result.

4.3. Strong convergence of truncations. To continue our proof of Theorem 3.1 we need the following proposition.

Proposition 4.3. Fix any truncation level $\gamma>0$. As $\varepsilon \rightarrow 0$, we have

$$
\limsup _{\varepsilon \rightarrow 0} \sum_{l=1}^{\mathrm{N}} \int_{\Omega}\left|\frac{\partial T_{\gamma}\left(u_{\varepsilon}\right)}{\partial x_{l}}\right|^{p_{l}(x)} d x \leq \sum_{l=1}^{\mathrm{N}} \int_{\Omega}\left|\frac{\partial T_{\gamma}(u)}{\partial x_{l}}\right|^{p_{l}(x)} d x
$$

and

$$
T_{\gamma}\left(u_{\varepsilon}\right) \rightarrow T_{\gamma}(u) \text { strongly in } \bigcap_{l=1}^{N} W_{0}^{1, p_{l}(\cdot)}(\Omega) .
$$

Proof. We start by showing how (4.30) can be deduced from (4.29).

Proof of (4.30). We recall the following well-known inequalities, which hold for any two real vectors $a, b$ and a real $p$ :

$$
\left(a|a|^{p-2}-b|b|^{p-2}\right)(a-b) \geq c(p) \begin{cases}|a-b|^{p}, & \text { if } p \geq 2 \\ \frac{|a-b|^{2}}{(|a|+|b|)^{2-p}}, & \text { if } 1<p<2,\end{cases}
$$

where $c(p)=2^{2-p}$ when $p \geq 2$ and $c(p)=p-1$ when $1<p<2$. 
Therefore we have for $p_{l}(\cdot) \geq 2$ for $l=1, \ldots, N$,

$$
\begin{aligned}
2^{2-p_{l}^{+}} \int_{\left\{x \in \Omega: p_{l}(x) \geq 2\right\}}\left|\frac{\partial T_{\gamma}\left(u_{\varepsilon}\right)}{\partial x_{l}}-\frac{\partial T_{\gamma}(u)}{\partial x_{l}}\right|^{p_{l}(x)} d x \\
\leq \int_{\Omega}\left(\left.\left|\frac{\partial T_{\gamma}\left(u_{\varepsilon}\right)}{\partial x_{l}}\right|\right|^{p_{l}(x)-2} \frac{\partial T_{\gamma}\left(u_{\varepsilon}\right)}{\partial x_{l}}-\left|\frac{\partial T_{\gamma}(u)}{\partial x_{l}}\right|^{p_{l}(x)-2} \frac{\partial T_{\gamma}(u)}{\partial x_{l}}\right) \\
\quad \times\left(\frac{\partial T_{\gamma}\left(u_{\varepsilon}\right)}{\partial x_{l}}-\frac{\partial T_{\gamma}(u)}{\partial x_{l}}\right) d x=: E(\varepsilon) .
\end{aligned}
$$

On the set where $1<p_{l}(\cdot)<2$ for $l=1, \ldots, N$, we employ (4.31) as follows:

$$
\begin{aligned}
& \int_{\left\{x \in \Omega: 1<p_{l}(x)<2\right\}}\left|\frac{\partial T_{\gamma}\left(u_{\varepsilon}\right)}{\partial x_{l}}-\frac{\partial T_{\gamma}(u)}{\partial x_{l}}\right|^{p_{l}(x)} d x \\
& \leq \int_{\left\{x \in \Omega: 1<p_{l}(x)<2\right\}} \frac{\left|\frac{\partial T_{\gamma}\left(u_{\varepsilon}\right)}{\partial x_{l}}-\frac{\partial T_{\gamma}(u)}{\partial x_{l}}\right|^{p_{l}(x)}}{\left(\left|\frac{\partial T_{\gamma}\left(u_{\varepsilon}\right)}{\partial x_{l}}\right|+\left|\frac{\partial T_{\gamma}(u)}{\partial x_{l}}\right|\right)^{\frac{p_{l}(x)\left(2-p_{l}(x)\right)}{2}}} \\
& \times\left(\left|\frac{\partial T_{\gamma}\left(u_{\varepsilon}\right)}{\partial x_{l}}\right|+\left|\frac{\partial T_{\gamma}(u)}{\partial x_{l}}\right|\right)^{\frac{p_{l}(x)\left(2-p_{l}(x)\right)}{2}} d x \\
& \leq 2\left\|\frac{\left|\frac{\partial T_{\gamma}\left(u_{\varepsilon}\right)}{\partial x_{l}}-\frac{\partial T_{\gamma}(u)}{\partial x_{l}}\right|^{p_{l}(x)}}{\left(\left|\frac{\partial T_{\gamma}\left(u_{\varepsilon}\right)}{\partial x_{l}}\right|+\left|\frac{\partial T_{\gamma}(u)}{\partial x_{l}}\right|\right)^{\frac{p_{l}(x)\left(2-p_{l}(x)\right)}{2}}}\right\|_{L^{2 / p_{l}(x)}(\Omega)} \\
& \times\left\|\left(\left|\frac{\partial T_{\gamma}\left(u_{\varepsilon}\right)}{\partial x_{l}}\right|+\left|\frac{\partial T_{\gamma}(u)}{\partial x_{l}}\right|\right)^{\frac{p_{l}(x)\left(2-p_{l}(x)\right)}{2}}\right\|_{L^{2 /\left(2-p_{l}(x)\right)}(\Omega)} \\
& \leq 2 \max \left\{\left(\int_{\Omega} \frac{\left|\frac{\partial T_{\gamma}\left(u_{\varepsilon}\right)}{\partial x_{l}}-\frac{\partial T_{\gamma}(u)}{\partial x_{l}}\right|^{2}}{\left(\left|\frac{\partial T_{\gamma}\left(u_{\varepsilon}\right)}{\partial x_{l}}\right|+\left|\frac{\partial T_{\gamma}(u)}{\partial x_{l}}\right|\right)^{2-p_{l}(x)}} d x\right)^{p_{l}^{-} / 2}\right. \\
& \left.,\left(\int_{\Omega} \frac{\left|\frac{\partial T_{\gamma}\left(u_{\varepsilon}\right)}{\partial x_{l}}-\frac{\partial T_{\gamma}(u)}{\partial x_{l}}\right|^{2}}{\left(\left|\frac{\partial T_{\gamma}\left(u_{\varepsilon}\right)}{\partial x_{l}}\right|+\left|\frac{\partial T_{\gamma}(u)}{\partial x_{l}}\right|\right)^{2-p_{l}(x)}} d x\right)^{p_{l}^{+} / 2}\right\} \\
& \times \max \left\{\left(\int_{\Omega}\left(\left|\frac{\partial T_{\gamma}\left(u_{\varepsilon}\right)}{\partial x_{l}}\right|+\left|\frac{\partial T_{\gamma}(u)}{\partial x_{l}}\right|\right)^{p_{l}(x)} d x\right)^{\left(2-p_{l}^{+}\right) / 2}\right. \\
& \left.,\left(\int_{\Omega}\left(\left|\frac{\partial T_{\gamma}\left(u_{\varepsilon}\right)}{\partial x_{l}}\right|+\left|\frac{\partial T_{\gamma}(u)}{\partial x_{l}}\right|\right)^{p_{l}(x)} d x\right)^{\left(2-p_{l}^{-}\right) / 2}\right\}
\end{aligned}
$$




$$
\begin{aligned}
\leq 2 \max \left\{\left(p_{l}^{-}-1\right)^{-p_{l}^{-} / 2}(E(\varepsilon))^{p_{l}^{-} / 2},\left(p_{l}^{-}-1\right)^{-p_{l}^{+} / 2}(E(\varepsilon))^{p_{l}^{+} / 2}\right\} \\
\times \max \left\{\left(\int_{\Omega}\left(\left|\frac{\partial T_{\gamma}\left(u_{\varepsilon}\right)}{\partial x_{l}}\right|+\left|\frac{\partial T_{\gamma}(u)}{\partial x_{l}}\right|\right)^{p_{l}(x)} d x\right)^{\left(2-p_{l}^{+}\right) / 2}\right. \\
\left.,\left(\int_{\Omega}\left(\left|\frac{\partial T_{\gamma}\left(u_{\varepsilon}\right)}{\partial x_{l}}\right|+\left|\frac{\partial T_{\gamma}(u)}{\partial x_{l}}\right|\right)^{p_{l}(x)} d x\right)^{\left(2-p_{l}^{-}\right) / 2}\right\} .
\end{aligned}
$$

Since $T_{\gamma}\left(u_{\varepsilon}\right)$ is bounded in $\bigcap_{l=1}^{N} W_{0}^{1, p_{l}(\cdot)}(\Omega)$ and using (4.27) and (4.29), it follows that $E(\varepsilon) \rightarrow 0$ as $\varepsilon \rightarrow 0$. Hence, sending $\varepsilon \rightarrow 0$ in (4.32) and (4.33) yields

$$
\lim _{\varepsilon \rightarrow 0} \int_{\Omega}\left|\frac{\partial T_{\gamma}\left(u_{\varepsilon}\right)}{\partial x_{l}}-\frac{\partial T_{\gamma}(u)}{\partial x_{l}}\right|^{p_{l}(x)} d x=0
$$

which proves (4.30).

Proof of (4.29). We substitute $\varphi=S_{n}^{\prime}\left(u_{\varepsilon}\right) V_{\varepsilon}$ in (4.2), where

$$
V_{\varepsilon}=T_{\gamma}\left(u_{\varepsilon}\right)-T_{\gamma}(u)
$$

The result is

$$
J_{\varepsilon, n}^{1}+J_{\varepsilon, n}^{2}+J_{\varepsilon, n}^{3}=J_{\mathcal{\varepsilon}, n}^{4}
$$

where

$$
\begin{aligned}
& J_{\varepsilon, n}^{1}=\int_{\Omega} S_{n}^{\prime}\left(u_{\varepsilon}\right) A\left(x, \nabla u_{\varepsilon}\right) \cdot \nabla V_{\varepsilon} d x, \\
& J_{\varepsilon, n}^{2}=\int_{\Omega} S_{n}^{\prime \prime}\left(u_{\varepsilon}\right) A\left(x, \nabla u_{\varepsilon}\right) \cdot \nabla u_{\varepsilon} V_{\varepsilon} d x, \\
& J_{\varepsilon, n}^{3}=\int_{\Omega} g\left(x, u_{\varepsilon}\right) S_{n}^{\prime}\left(u_{\varepsilon}\right) V_{\varepsilon} d x, \\
& J_{\varepsilon, n}^{4}=\int_{\Omega} f_{\varepsilon} S_{n}^{\prime}\left(u_{\varepsilon}\right) V_{\varepsilon} d x .
\end{aligned}
$$

Our goal is to pass to the limit in (4.35)-(4.36) as $\varepsilon$ and $n$ goes to 0 and $\infty$, respectively, with the truncation level $\gamma$ kept fixed!

Limit of $J_{\varepsilon, n}^{2}$. Using Lemma 4.2 and as $\operatorname{supp} S_{n}^{\prime} \subset[-(n+1),-n] \cup[n, n+1]$, we obtain for any $n \geq 2$

$$
\limsup _{n \rightarrow \infty} \limsup _{\varepsilon \rightarrow 0}\left|J_{\varepsilon, n}^{2}\right| \leq C \limsup _{n \rightarrow \infty} \limsup _{\varepsilon \rightarrow 0} \int_{\left\{n \leq\left|u_{\varepsilon}\right| \leq n+1\right\}}\left(1+\left|\frac{\partial u_{\varepsilon}}{\partial x_{l}}\right|^{p_{l}(x)}\right) d x=0,
$$

where $\mathrm{C}$ is a constant independent of $n$.

Limit of $J_{\varepsilon, n}^{3}$ and $J_{\varepsilon, n}^{3}$. In view of Lebesgue's convergence theorem implies that for any $n \geq 2$

$$
\lim _{\mathcal{\varepsilon} \rightarrow 0} J_{\mathcal{\varepsilon}, n}^{3}=0 \text { and } \lim _{\varepsilon \rightarrow 0} J_{\mathcal{\varepsilon}, n}^{4}=0 .
$$


Now we can pass to the limsup in (4.35) when $\varepsilon \rightarrow 0$ and $n \rightarrow \infty$, respectively. The result is that for any $\gamma \geq 0$

$$
\limsup _{n \rightarrow \infty} \limsup _{\varepsilon \rightarrow 0} \int_{\Omega} S_{n}^{\prime}\left(u_{\varepsilon}\right)\left|\frac{\partial u_{\varepsilon}}{\partial x_{l}}\right|^{p_{l}(x)-2} \frac{\partial u_{\varepsilon}}{\partial x_{l}} \frac{\partial V_{\varepsilon}}{\partial x_{l}} d x \leq 0 .
$$

By the definition of $S_{n}$ (see Subsection 3), we have for any $n \geq \gamma$

$$
S_{n}^{\prime}\left(u_{\varepsilon}\right)\left|\frac{\partial u_{\varepsilon}}{\partial x_{l}}\right|^{p_{l}(x)-2} \frac{\partial u_{\varepsilon}}{\partial x_{l}} \frac{\partial T_{\gamma}\left(u_{\varepsilon}\right)}{\partial x_{l}}=\left|\frac{\partial T_{\gamma}\left(u_{\varepsilon}\right)}{\partial x_{l}}\right|^{p_{l}(x)} .
$$

Using this and the definition of $V_{\varepsilon}$, we deduce from (4.39)

$$
\limsup _{\varepsilon \rightarrow 0} \int_{\Omega}\left|\frac{\partial T_{\gamma}\left(u_{\varepsilon}\right)}{\partial x_{l}}\right|^{p_{l}(x)} d x \leq \underset{n \rightarrow \infty}{\limsup } \limsup _{\varepsilon \rightarrow 0} \int_{\Omega} S_{n}^{\prime}\left(u_{\varepsilon}\right)\left|\frac{\partial u_{\varepsilon}}{\partial x_{l}}\right|^{p_{l}(x)-2} \frac{\partial u_{\varepsilon}}{\partial x_{l}} \frac{\partial T_{\gamma}\left(u_{\varepsilon}\right)}{\partial x_{l}} d x
$$

By the definition of $S_{n}$, we obtain

$$
S_{n}^{\prime}\left(u_{\varepsilon}\right)\left|\frac{\partial u_{\varepsilon}}{\partial x_{l}}\right|^{p_{l}(x)-2} \frac{\partial u_{\varepsilon}}{\partial x_{l}}=S_{n}^{\prime}\left(u_{\varepsilon}\right)\left|\frac{\partial T_{n+1}\left(u_{\varepsilon}\right)}{\partial x_{l}}\right|^{p_{l}(x)-2} \frac{\partial T_{n+1}\left(u_{\varepsilon}\right)}{\partial x_{l}} \quad \text { a.e. in } \Omega \text {. }
$$

Using the a.e. convergence of $S_{n}^{\prime}\left(u_{\varepsilon}\right)$ to $S_{n}^{\prime}(u)$ as $\varepsilon \rightarrow 0$, the boundedness of $S_{n}^{\prime}$, and the weak convergence of $T_{n+1}\left(u_{\varepsilon}\right)$ to $T_{n+1}(u)$ in $\bigcap_{l=1}^{N} W_{0}^{1, p_{l}(\cdot)}(\Omega)$ as $\varepsilon \rightarrow 0$, together with an application of the usual Minty argument, we obtain

$$
S_{n}^{\prime}\left(u_{\varepsilon}\right)\left|\frac{\partial u_{\varepsilon}}{\partial x_{l}}\right|^{p_{l}(x)-2} \frac{\partial u_{\varepsilon}}{\partial x_{l}} \rightarrow S_{n}^{\prime}(u)\left|\frac{\partial T_{n+1}(u)}{\partial x_{l}}\right|^{p_{l}(x)-2} \frac{\partial T_{n+1}(u)}{\partial x_{l}}
$$

weakly in $L^{p^{\prime}(\cdot)}(\Omega)$.

Sending $\varepsilon \rightarrow 0$ in (4.40), we deduce for any $n \geq \gamma$

$$
\begin{aligned}
\limsup _{\varepsilon \rightarrow 0}\left|\frac{\partial T_{\gamma}\left(u_{\varepsilon}\right)}{\partial x_{l}}\right|^{p_{l}(x)} d x & \leq \limsup _{n \rightarrow \infty} \limsup _{\varepsilon \rightarrow 0} \int_{\Omega} S_{n}^{\prime}\left(u_{\varepsilon}\right)\left|\frac{\partial u_{\varepsilon}}{\partial x_{l}}\right|^{p_{l}(x)-2} \frac{\partial u_{\varepsilon}}{\partial x_{l}} \frac{\partial T_{\gamma}\left(u_{\varepsilon}\right)}{\partial x_{l}} d x \\
& =\limsup _{n \rightarrow \infty} \int_{\Omega} S_{n}^{\prime}(u)\left|\frac{\partial T_{n+1}(u)}{\partial x_{l}}\right|^{p_{l}(x)-2} \frac{\partial T_{n+1}(u)}{\partial x_{l}} \frac{\partial T_{n+1}(u)}{\partial x_{l}} d x \\
& =\int_{\Omega}\left|\frac{\partial T_{n+1}(u)}{\partial x_{l}}\right|^{p_{l}(x)} d x .
\end{aligned}
$$

The proof of Proposition 4.3 is completed.

4.4. Concluding the proof of Theorem 3.1. Let $S \in C^{\infty}(\mathbb{R})$ be such that $\operatorname{supp} S^{\prime} \subset[-M, M]$ for some $M>0$. Multiplying (3.1) by $S^{\prime}\left(u_{\varepsilon}\right)$ (where $u$ and $f$ are replaced by $u_{\varepsilon}$ and $f_{\varepsilon}$ respectively) yields

$$
-\operatorname{div}\left(S^{\prime}\left(u_{\varepsilon}\right) A\left(x, \nabla u_{\varepsilon}\right)\right)+S^{\prime \prime}\left(u_{\varepsilon}\right) A\left(x, \nabla u_{\varepsilon}\right) \cdot \nabla u_{\varepsilon}=-S^{\prime}\left(u_{\varepsilon}\right) g\left(x, u_{\varepsilon}\right)+f_{\varepsilon} S^{\prime}\left(u_{\varepsilon}\right) \quad \text { in } \mathcal{D}^{\prime}(\Omega) .
$$


In the following we pass to the limit $\varepsilon \rightarrow 0$ (in the sense of distributions) in each of the terms in (4.42). Thanks to (4.27) and Proposition 4.2, it is easy to pass to the limit in the right hand side term.

Let us consider the first term.

$$
S^{\prime}\left(u_{\varepsilon}\right) A\left(x, \nabla u_{\varepsilon}\right)=S^{\prime}\left(u_{\varepsilon}\right) A\left(x, \nabla T_{M}\left(u_{\varepsilon}\right)\right),
$$

and, because of (4.30),

$$
S^{\prime}\left(u_{\varepsilon}\right) A\left(x, \nabla T_{M}\left(u_{\varepsilon}\right)\right) \rightarrow S^{\prime}(u) A\left(x, \nabla T_{M}(u)\right) \text { strongly in } L^{p(\cdot)^{\prime}}(\Omega) .
$$

Since

$$
S^{\prime}(u) A\left(x, \nabla T_{M}(u)\right)=S^{\prime}(u) A(x, \nabla u),
$$

this concludes passing to the limit in the first term in (4.42).

Let us now study the second term. Clearly, since supp $S^{\prime \prime} \subset[-M, M]$, we have

$$
S^{\prime \prime}\left(u_{\varepsilon}\right) A\left(x, \nabla u_{\varepsilon}\right) \cdot \nabla u_{\varepsilon}=S^{\prime \prime}\left(u_{\varepsilon}\right) A\left(x, \nabla T_{M}\left(u_{\varepsilon}\right)\right) \cdot \nabla T_{M}\left(u_{\varepsilon}\right) \quad \text { a.e. in } \Omega .
$$

Thanks to (4.30), the fact that $S^{\prime \prime}\left(u_{\varepsilon}\right)$ converges to $S^{\prime \prime}(u)$ a.e. in $\Omega$, and the boundedness of $S^{\prime \prime}$, the following convergence is true:

$S^{\prime \prime}\left(u_{\varepsilon}\right) A\left(x, \nabla T_{M}\left(u_{\varepsilon}\right)\right) \cdot \nabla T_{M}\left(u_{\varepsilon}\right) \rightarrow S^{\prime \prime}(u) A\left(x, \nabla T_{M}(u)\right) \cdot \nabla T_{M}(u)$ strongly in $L^{1}(\Omega)$ as $\varepsilon \rightarrow 0$.

This concludes the treatment of the third term in (4.42), as

$$
S^{\prime \prime}(u) A\left(x, \nabla T_{M}(u)\right) \cdot \nabla T_{M}(u)=S^{\prime \prime}(u) A(x, \nabla u) \cdot \nabla u .
$$

Let us derive the entropy formulation for the regularized sequence $u_{\varepsilon}$. Let $\phi \in C_{c}(\Omega)$ and consider $\varphi=T_{\gamma}\left(u_{\varepsilon}-\phi\right)$ as test function in (4.2). The result is

$$
\int_{\Omega} A\left(x, \nabla u_{\varepsilon}\right) \cdot \nabla T_{\gamma}\left(u_{\varepsilon}-\phi\right) d x+\int_{\Omega} g\left(x, u_{\varepsilon}\right) T_{\gamma}\left(u_{\varepsilon}-\phi\right)=\int_{\Omega} f_{\varepsilon} T_{\gamma}\left(u_{\varepsilon}-\phi\right) d x .
$$

Using the strong convergence of $f_{\varepsilon}$ and $g\left(\cdot, u_{\varepsilon}\right),(4.27)$ and Proposition 4.3 , we can pass to the limits as $\varepsilon \rightarrow 0$ in (4.43) to deduce

$$
\int_{\Omega} A(x, \nabla u) \cdot \nabla T_{\gamma}(u-\phi) d x+\int_{\Omega} g(x, u) T_{\gamma}(u-\phi)=\int_{\Omega} f T_{\gamma}(u-\phi) d x,
$$

for all $\phi \in C_{c}(\Omega)$.

\section{Uniqueness of renormalized solutions}

The purpose of this section is to adapt some of the existing uniqueness results for elliptic equations with variable exponents and $L^{1}$ data (see e.g. [27]).

We have the following theorem.

Theorem 5.1. The renormalized solution of problem (3.1) is unique and the comparison principle holds. 
Proof. In what follows, we let $u, v$ be two renormalized solutions in the sense of Definition 3.2 with data $f, g$, respectively.

For $\mu>0$, define the function $S_{\mu}$ in $W^{2, \infty}(\mathbb{R})$ defined by $S_{\mu}(0)=0$ and

$$
S_{\mu}^{\prime}(r)= \begin{cases}1, & \text { for }|r| \leq \mu, \\ \mu+1-|r|, & \text { for } \mu \leq|r| \leq \mu+1, \\ 0, & \text { for }|r| \geq \mu+1 .\end{cases}
$$

Note that supp $S_{\mu}^{\prime} \subset[-\mu-1, \mu+1]$ and $S_{\mu}$ in $W^{2, \infty}(\mathbb{R})$, thus we can take $S=S_{\mu}$ in (3.11). The function $T_{\gamma}\left(\left(S_{\mu}(u)-S_{\mu}(v)\right)^{+}\right)$belongs to $\bigcap_{l=1}^{N} W_{0}^{1, p(\cdot)}(\Omega) \cap L^{\infty}(\Omega)$, and thus it can be used as a test function in (3.11).

Now, we use $T_{\gamma}\left(\left(S_{\mu}(u)-S_{\mu}(v)\right)^{+}\right)$as a test function in (3.11) for $u$ and for $v$ with data $f$ and $g$, respectively, and then subtract the resulting equations. The result is

$$
A_{1}^{\mu, \gamma}+A_{2}^{\mu, \gamma}+A_{3}^{\mu, \gamma}+A_{4}^{\mu, \gamma}=\int_{\Omega}\left(f S_{\mu}^{\prime}(u)-g S_{\mu}^{\prime}(v)\right) T_{\gamma}\left(\left(S_{\mu}(u)-S_{\mu}(v)\right)^{+}\right) d x,
$$

where

$$
\begin{aligned}
A_{1}^{\mu, \gamma} & =\int_{\Omega}\left[S_{\mu}^{\prime}(u) A(x, \nabla u)-S_{\mu}^{\prime}(v) A(x, \nabla v)\right] \cdot \nabla T_{\gamma}\left(\left(S_{\mu}(u)-S_{\mu}(v)\right)^{+}\right) d x, \\
A_{2}^{\mu, \gamma} & =\int_{\Omega} S_{\mu}^{\prime \prime}(u) T_{\gamma}\left(\left(S_{\mu}(u)-S_{\mu}(v)\right)^{+}\right) A(x, \nabla u) \cdot \nabla u d x, \\
A_{3}^{\mu, \gamma} & =-\int_{\Omega} S_{\mu}^{\prime \prime}(v) T_{\gamma}\left(\left(S_{\mu}(u)-S_{\mu}(v)\right)^{+}\right) A(x, \nabla v) \cdot \nabla v d x, \\
A_{4}^{\mu, \gamma} & =-\int_{\Omega}\left(S_{\mu}^{\prime}(u) g(x, u)-S_{\mu}^{\prime}(v) g(x, v)\right) T_{\gamma}\left(\left(S_{\mu}(u)-S_{\mu}(v)\right)^{+}\right) d x .
\end{aligned}
$$

Observe that

$$
\begin{aligned}
A_{1}^{\mu, \gamma}= & \int_{\Omega}\left(A\left(x, \nabla S_{\mu}(u)\right)-A\left(x, \nabla S_{\mu}(v)\right)\right) \cdot \nabla T_{\gamma}\left(\left(S_{\mu}(u)-S_{\mu}(v)\right)^{+}\right) d x \\
& +\int_{\Omega}\left(S_{\mu}^{\prime}(u) A(x, \nabla u)-A\left(x, \nabla S_{\mu}(u)\right)\right) \cdot \nabla T_{\gamma}\left(\left(S_{\mu}(u)-S_{\mu}(v)\right)^{+}\right) d x \\
& -\int_{\Omega}^{2}\left(S_{\mu}^{\prime}(v) A(x, \nabla v)-A\left(x, \nabla S_{\mu}(v)\right)\right) \cdot \nabla T_{\gamma}\left(\left(S_{\mu}(u)-S_{\mu}(v)\right)^{+}\right) d x \\
:=\quad & A_{1,1}^{\mu, \gamma}+A_{1,2}^{\mu, \gamma}+A_{1,3}^{\mu, \gamma} .
\end{aligned}
$$

By the definition of $S_{\mu}$, we obtain from (3.3)

$$
\begin{aligned}
\left|A_{1,2}^{\mu, \gamma}\right| & \leq C \sum_{1=1}^{\mathrm{N}}\left(\int_{\{\mu \leq|u| \leq \mu+1\}}\left(1+\left|\frac{\partial u}{\partial x_{l}}\right|^{p_{l}(x)}\right) d x+\int_{\{\mu-\gamma \leq|u| \leq \mu+1\}}\left(1+\left|\frac{\partial v}{\partial x_{l}}\right|^{p_{l}(x)}\right) d x\right) \\
& \leq \varepsilon_{1}(\mu),
\end{aligned}
$$

for any $\mu>\gamma>0$, where $\varepsilon_{1}(\mu)$ tends to zero as $\mu \rightarrow \infty$.

Along the same lines we obtain

$$
\left|A_{1,3}^{\mu, \gamma}\right| \leq \varepsilon_{2}(\mu)
$$


where $\varepsilon_{2}(\mu)$ tends to zero as $\mu \rightarrow \infty$. Next, observe that $\left|S_{\mu}^{\prime \prime}(r)\right|=\mathbf{1}_{\{\mu \leq|r| \leq \mu+1\}}$. Using this to deduce

$$
\lim _{\mu \rightarrow \infty}\left|A_{2}^{\mu, \gamma}\right|=\lim _{\mu \rightarrow \infty}\left|A_{3}^{\mu, \gamma}\right|=0 .
$$

We let $\mu_{0} \geq 0$ such that $\mu \geq \mu_{0}$. Observe that

$$
A_{1,1}^{\mu, \gamma} \geq \int_{\{0 \leq u-v \leq \gamma\} \cap\left\{|u|,|v| \leq \mu_{0}\right\}}(A(x, \nabla u)-A(x, \nabla v)) \cdot \nabla(u-v) d x .
$$

Using all the previous calculations, together with the facts that, as $\mu \rightarrow \infty$,

$$
\begin{aligned}
& \left(S_{\mu}^{\prime}(u) g(x, u)-S_{\mu}^{\prime}(v) g(x, v)\right) T_{\gamma}\left(\left(S_{\mu}(u)-S_{\mu}(v)\right)^{+}\right) \rightarrow(g(x, u)-g(x, v)) T_{\gamma}\left((u-v)^{+}\right), \\
& \left(f S_{\mu}^{\prime}(u)-g S_{\mu}^{\prime}(v)\right) T_{\gamma}\left(\left(S_{\mu}(u)-S_{\mu}(v)\right)^{+}\right) \rightarrow(f-g) T_{\gamma}\left((u-v)^{+}\right),
\end{aligned}
$$

strongly in $L^{1}(\Omega)$, it follows, by sending $\mu \rightarrow \infty$ in (5.2) that

$$
\int_{\{0 \leq u-v \leq \gamma\} \cap\left\{|u|,|v| \leq \mu_{0}\right\}}(A(x, \nabla u)-A(x, \nabla v)) \cdot \nabla(u-v) d x \leq \int_{\Omega}(f-g) T_{\gamma}\left((u-v)^{+}\right) d x .
$$

Supposing now $f \leq g$ a.e. in $\Omega$ in (5.5), we deduce

$$
\sum_{l=1}^{N} \int_{\{0 \leq u-v \leq \gamma\} \cap\left\{|u|,|v| \leq \mu_{0}\right\}}\left(\left|\frac{\partial u}{\partial x_{l}}\right|^{p_{l}(x)-2} \frac{\partial u}{\partial x_{l}}-\left|\frac{\partial v}{\partial x_{l}}\right|^{p_{l}(x)-2} \frac{\partial v}{\partial x_{l}}\right) \frac{\partial u_{\varepsilon}}{\partial x_{l}}(u-v) d x=0 .
$$

Sending $\gamma \rightarrow \infty$ in (5.6) and using the inequality (4.31), we obtain

$$
\frac{\partial T_{\mu_{0}}(u)}{\partial x_{l}}=\frac{\partial T_{\mu_{0}}(v)}{\partial x_{l}} \text { a.e. on the set }\{u>v\} \cap\left\{|u|,|v| \leq \mu_{0}\right\} \text {. }
$$

As $\mu_{0}$ is arbitrary, we get

$$
\frac{\partial u}{\partial x_{l}}=\frac{\partial v}{\partial x_{l}} \text { a.e. on the set }\{u>v\} \text {, i.e. } \nabla(u-v)^{+}=0 \text { a.e. on } \Omega \text {. }
$$

Since $(u-v)^{+} \in W_{0}^{1,1}(\Omega)$, we deduce

$$
(u-v)^{+}=0 \text { a.e. on the set } \Omega,
$$

i.e. $u \leq v$ a.e. on $\Omega$. The uniqueness assertion follows from this comparison principle.

\section{Comparison between entropy and renormalized solutions}

In this section, we extend a previous notion of entropy solutions and prove that they coincide with the renormalized solutions we prove the following theorem.

Theorem 6.1. Under hypotheses (3.2)-(3.7) hold and the corresponding exponents functions $p_{1}(\cdot), \ldots$, $p_{N}(\cdot)$ and $s(\cdot)$ are restricted as in (1.2). Then, a function is an entropy solution of (3.1) if and only if it is a renormalized solution of (3.1). 
Proof. We prove now that the entropy solution $u$ satisfies all the other properties of the renormalized solution. First we observe that the entropy solution $u$ in Theorem 6.1 satisfies the requirements that $T_{\gamma}\left(u_{\varepsilon}\right) \in W_{0}^{1, p(x)}(\Omega)$ for given $\gamma>0$. Inserting $\varphi=T_{1}\left(u_{\varepsilon}-T_{\gamma}\left(u_{\varepsilon}\right)\right)$ into (4.2) gives

$$
\int_{\left\{\gamma \leq\left|u_{\varepsilon}\right| \leq \gamma+1\right\}} A\left(x, \nabla u_{\varepsilon}\right) \cdot \nabla u_{\varepsilon} d x+\int_{\left\{\gamma \leq\left|u_{\varepsilon}\right| \leq \gamma+1\right\}} g\left(x, u_{\varepsilon}\right) u_{\varepsilon} d x=\int_{\Omega} f_{\varepsilon} T_{1}\left(u_{\varepsilon}-T_{\gamma}\left(u_{\varepsilon}\right)\right) d x .
$$

By (3.2), (3.5) and letting $\varepsilon \rightarrow 0$, we have

$$
\sum_{l=1}^{N} \int_{\{\gamma \leq|u| \leq \gamma+1\}}\left|\frac{\partial u}{\partial x_{l}}\right|^{p_{l}(x)} d x \leq \int_{\{\gamma \leq|u|\}}|f| d x,
$$

then, we deduce the renormalized condition, that is,

$$
\lim _{\gamma \rightarrow \infty} \sum_{l=1}^{N} \int_{\{\gamma \leq|u| \leq \gamma+1\}}\left|\frac{\partial u}{\partial x_{l}}\right|^{p_{l}(x)} d x=0 .
$$

Let $\left(u_{\varepsilon}\right)_{\varepsilon}$ be a sequence constructed in (4.2), which satisfies the requirement that $T_{\gamma}\left(u_{\varepsilon}\right)$ strongly converges to $T_{\gamma}(u)$ in $W_{0}^{1, p(x)}(\Omega)$ for every $\gamma>0$.

Let $S \in C^{\infty}(\mathbb{R})$ be such that supp $S^{\prime} \in[-M, M]$ for some $M>0$. For every $\varphi \in C_{0}^{\infty}(\Omega)$, taking $S^{\prime}\left(u_{\varepsilon}\right) \varphi \in W_{0}^{1, p_{l}(x)}(\Omega)$ as a test function in (4.2). We get

$$
\int_{\Omega} A\left(x, \nabla u_{\varepsilon}\right)\left(S^{\prime \prime}\left(u_{\varepsilon}\right) \varphi \nabla u_{\varepsilon}+S^{\prime}\left(u_{\varepsilon}\right) \nabla \varphi\right) d x+\int_{\Omega} g\left(x, \nabla u_{\varepsilon}\right) S^{\prime}\left(u_{\varepsilon}\right) \varphi d x=\int_{\Omega} f_{\varepsilon} S^{\prime}\left(u_{\varepsilon}\right) \varphi d x .
$$

We start with the first integral in (6.2), we have

$$
\int_{\Omega} A\left(x, \nabla u_{\varepsilon}\right)\left(S^{\prime \prime}\left(u_{\varepsilon}\right) \varphi \nabla u_{\varepsilon}+S^{\prime}\left(u_{\varepsilon}\right) \nabla \varphi\right) d x=\int_{\Omega} A\left(x, \nabla T_{\gamma}\left(u_{\varepsilon}\right)\right)\left(S^{\prime \prime}\left(u_{\varepsilon}\right) \varphi \nabla T_{\gamma}\left(u_{\varepsilon}\right)+S^{\prime}\left(u_{\varepsilon}\right) \nabla \varphi\right) d x,
$$

from (3.3), we have $A\left(x, \nabla T_{\gamma}\left(u_{\varepsilon}\right)\right)$ is bounded in $\left(L^{p^{\prime}(x)}(\Omega)\right)^{N}$, then

$$
A\left(x, \nabla T_{\gamma}\left(u_{\varepsilon}\right)\right) \rightarrow A\left(x, \nabla T_{\gamma}(u)\right) \text { a.e. in } \Omega \text {. }
$$

Hence, we get

$$
A\left(x, \nabla T_{\gamma}\left(u_{\varepsilon}\right)\right) \rightarrow A\left(x, \nabla T_{\gamma}(u)\right) \quad \text { in }\left(L^{p^{\prime}(x)}(\Omega)\right)^{N},
$$

and since

$$
S^{\prime \prime}\left(u_{\varepsilon}\right) \varphi \nabla T_{\gamma}\left(u_{\varepsilon}\right)+S^{\prime}\left(u_{\varepsilon}\right) \nabla \varphi \longrightarrow S^{\prime \prime}(u) \varphi \nabla T_{\gamma}(u)+S^{\prime}(u) \nabla \varphi \quad \text { in }\left(L^{p(x)}(\Omega)\right)^{N},
$$

we deduce that

$$
\begin{gathered}
\lim _{\varepsilon \rightarrow 0} \int_{\Omega} A\left(x, \nabla T_{\gamma}\left(u_{\varepsilon}\right)\right)\left(S^{\prime \prime}\left(u_{\varepsilon}\right) \varphi \nabla T_{\gamma}\left(u_{\varepsilon}\right)+S^{\prime}\left(u_{\varepsilon}\right) \nabla \varphi\right) d x \\
=\int_{\Omega} A\left(x, \nabla T_{\gamma}(u)\right)\left(S^{\prime \prime}(u) \varphi \nabla T_{\gamma}(u)+S^{\prime}(u) \nabla \varphi\right) d x \\
=\int_{\Omega} A(x, \nabla u)\left(S^{\prime \prime}(u) \varphi \nabla u+S^{\prime}(u) \nabla \varphi\right) d x .
\end{gathered}
$$


We next examine the second term on the right-hand side of (6.2), we have $S\left(u_{\varepsilon}\right) \varphi \rightarrow S\left(u_{\varepsilon}\right) \varphi$ weak-* in $L^{\infty}(\Omega)$, then

$$
\int_{\Omega} g\left(x, u_{\varepsilon}\right) S^{\prime}\left(u_{\varepsilon}\right) \varphi d x \longrightarrow \int_{\Omega} g(x, u) S^{\prime}(u) \varphi d x
$$

and

$$
\int_{\Omega} f_{\varepsilon} S^{\prime}\left(u_{\varepsilon}\right) \varphi d x \longrightarrow \int_{\Omega} f S^{\prime}(u) \varphi d x
$$

By combining (6.2)-(6.4), we deduce that

$$
\int_{\Omega} A(x, \nabla u)\left(S^{\prime \prime}(u) \varphi \nabla u+S^{\prime}(u) \nabla \varphi\right) d x+\int_{\Omega} g(x, \nabla u) S^{\prime}(u) \varphi d x=\int_{\Omega} f S^{\prime}(u) \varphi d x,
$$

which is (3.11) in Definition 3.2. Then, we conclude that $u$ is a renormalized solution to problem (3.1). Thanks to uniqueness of renormalized solutions see Section 5, which concludes the proof of the Theorem 6.1.

\section{References}

[1] A. Aissaoui Fqayeh, A. Benkirane and M. El Moumni. Entropy solutions for strongly nonlinear unilateral parabolic inequalities in Orlicz-Sobolev spaces. Applicationes Mathematicae, 41,2-3 , (2014)pp: 185-193 .

[2] A. Aissaoui Fqayeh, A. Benkirane, M. El Moumni and A. Youssfi. Existence of renormalized solutions for some strongly nonlinear elliptic equations in Orlicz spaces. Georgian Math. J. Volume 22, Number 3,(2015), pp:305-321.

[3] Y. Akdim, M. Belayachi and M. El Moumni. $L^{\infty}$-bounds of solutions for strongly nonlinear elliptic problems with two lower order terms. Anal. Theory Appl., Vol. 33, No. 1 ,(2017) pp. 46- 58 .

[4] Y. Akdim, A. Benkirane, S. M. Douiri and M. El Moumni. On a quasilinear degenerated elliptic unilateral problems with $L^{1}$ data. Rend. Circ. Mat. Palermo, II. Ser (2018)67:43-57 .

[5] Y. Akdim, A. Benkirane and M. El Moumni. Solutions of nonlinear elliptic problems with lower order terms. Annals of Functional Analysis (AFA), Volume 6, Number 1, (2015), pp: 34-53.

[6] Y. Akdim, A. Benkirane and M. El Moumni. Existence results for nonlinear elliptic problems with lower order terms. International Journal of Evolution Equations (IJEE), Volume 8, Number 4 , (2014) pp: 1-20.

[7] Y. Akdim, A. Benkirane, M. El Moumni and A. Fri. Strongly nonlinear variational parabolic initial-boundary value problems. Annals of the University of Craiova - Mathematics and Computer Science Series. Volume 41, Number 2(2014), pp: 1-13.

[8] Y. Akdim, A. Benkirane, M. El Moumni and H. Redwane. Existence of renormalized solutions for nonlinear parabolic equations. Journal of Partial Differential Equations (JPDE), Volume 27, Number 1, (2014)pp: 28-49.

[9] Y. Akdim, A. Benkirane, M. El Moumni and H. Redwane. Existence of renormalized solutions for strongly nonlinear parabolic problems with measure data. Georgian Math. J. Volume 23, Issue 3, (2016)pp: 303321.

[10] Y. Akdim, M. El Moumni and A. Salmani. Existence Results for Nonlinear Anisotropic Elliptic Equation. Adv. Sci. Technol. Eng. Syst. J. 2(5), (2017)160-166.

[11] F. Andreu, J. M. Mazón, S. Segura de León and J. Toledo. Existence and uniqueness for a degenerate parabolic equation with $L^{1}$-data. Trans. Amer. Math. Soc. 351 (1999) no.1, 285-306.

[12] M. Bendahmane, K.H. Karlsen and M. Saad. Nonlinear anisotropic elliptic and parabolic equations with variable exponents and $L^{1}$-data. Comm. Pure and Appl. Anal. 12 (2013) no.3, 1201-1220.

[13] M. Bendahmane, M. Langlais and M. Saad. On some anisotropic reaction-diffusion systems with $L^{1}$-data modeling the propagation of an epidemic disease. Nonlinear Anal., 54(4) (2003), 617-636.

[14] M. Bendahmane and P. Wittbold. Renormalized solutions for nonlinear elliptic equations with variable exponents and $L^{1}$-data. Nonlinear Analysis TMA 70 (2009) no.2, 567-583. 
[15] Ph. Bénilan, L. Boccardo, T. Gallouët, R. Gariepy, M. Pierre, and J. L. Vázquez. An $L^{1}$-theory of existence and uniqueness of solutions of nonlinear elliptic equations. Ann. Scuola Norm. Sup. Pisa Cl. Sci. (4), 22(2) (1995), 241-273.

[16] M. B. Benboubker, H. Chrayteh, M. El Moumni and H. Hjiaj. Entropy and renormalized solutions for nonlinear elliptic problem involving variable exponent and measure data. Acta Mathematica Sinica, English Series. Volume 31, Number 1,(2015) , pp: 151-169.

[17] A. Benkirane, Y. El Hadfi and M. El Moumni. Renormalized solutions for nonlinear parabolic problems with $L^{1}$ - data in orlicz-sobolev spaces. Bulletin of Parana's Mathematical Society (3s.) Volume 35, Number 1, (2017), pp: $57-84$.

[18] A. Benkirane, B. El Haji and M. El Moumni. On the existence of solutions for degenerate parabolic equations with singular terms. Pure and Applied Mathematics Quaterly. Volume 14, Number 3-4, (2018), 591-606.

[19] A. Benkirane, M. El Moumni and A. Fri. An approximation of Hedberg's type in Sobolev spaces with variable exponent and application. Chinese Journal of Mathematics, Volume 2014, Article ID 549051, (2014)7 pages .

[20] A. Benkirane, M. El Moumni and A. Fri. Renormalized solution for strongly nonlinear elliptic problems with lower order terms and $L^{1}$-data. Izvestiya RAN: Ser. Mat. (2017)81:3 3-20 .

[21] A. Benkirane, M. El Moumni and K. Kouhaila. Solvability of strongly nonlinear elliptic variational problems in weighted Orlicz-Sobolev spaces. SeMA Journal (2020) 77:119-142.

[22] D. Blanchard and F. Murat. Renormalised solutions of nonlinear parabolic problems with $L^{1}$ data: existence and uniqueness. Proc. Roy. Soc. Edinburgh Sect. A, 127(6) (1997), 1137-1152.

[23] L. Boccardo, J. I. Diaz, D. Giachetti, and F. Murat. Existence of a solution for a weaker form of a nonlinear elliptic equation. In Recent advances in nonlinear elliptic and parabolic problems (Nancy, 1988), volume 208 of Pitman Res. Notes Math. Ser., pages 229-246. Longman Sci. Tech., Harlow, 1989.

[24] L. Boccardo, T. Gallouët, and P. Marcellini. Anisotropic equations in L ${ }^{1}$. Differential Integral Equations, 9(1) (1996), 209-212.

[25] M. Bourahma and J. Bennouna and M. El Moumni. $L^{\infty}$-Bounds of solutions for a nonlinear degenerate elliptic equations in Musielak spaces. Moroccan J. of Pure and Appl. Anal. (MJPAA) Volume 6(1), 2020, Pages 16-33.

[26] Y. Chen, S. Levine and M. Rao. Variable exponent, linear growth functionals in image restoration. SIAM J. Appl. Math. 66 (2006), 1383-1406.

[27] G. Dal Maso, F. Murat, L. Orsina and A. Prignet. Renormalized solutions of elliptic equations with general measure data. Ann. Sc. Norm. Super. Pisa Cl. Sci.(4) 28 (4) (1999), 741-808.

[28] R. J. DiPerna and P.-L. Lions. On the Cauchy problem for Boltzmann equations: global existence and weak stability. Ann. of Math. (2), 130(2) (1989), 321-366.

[29] B. El Haji, M. El Moumni and K. Kouhaila. On a nonlinear elliptic problems having large monotonocity with $L^{1}$-data in weighted Orlicz-Sobolev spaces. Moroccan J. of Pure and Appl. Anal. (MJPAA) Volume 5(1), (2019), Pages 104-116.

[30] M. El Moumni. Nonlinear elliptic equations without sign condition and $L^{1}$-data in Musielak-Orlicz-Sobolev spaces. Acta. Appl. Math. 159:95-117(2019).

[31] M. El Moumni. Renormalized solutions for strongly nonlinear elliptic problems with lower order terms and measure data in Orlicz-Sobolev spaces. Iran. J. Math. Sci. Inform. Vol 14, No 1, (2019)pp 95-119.

[32] M. El Moumni. Entropy solution for strongly nonlinear elliptic problems with lower order terms and $L^{1}$-data. Annals of the University of Craiova - Mathematics and Computer Science Series. Volume 40, Number 2 (2013), pp: 211-225.

[33] X.L. Fan, J. Shen and D. Zhao. Sobolev embedding theorems for spaces $W^{k, p(x)}(\Omega)$. J. Math. Anal. Appl. 262 (2001), 749-760.

[34] X.L. Fan and D. Zhao. On the spaces $L^{p(x)}(U)$ and $W^{m, p(x)}(U)$. J. Math. Anal. Appl. 263 (2001), 424-446.

[35] T. Harjulehto, P. Hästö, M. Koskenoja and S. Varonen. The Dirichlet energy integral and variable exponent Sobolev spaces with zero boundary values. Potential Anal. 25 (2006) no.3, 205-222.

[36] F. Li and H. Zhao. Anisotropic parabolic equations with measure data. J. Partial Differential Equations, 14(1) (2001), 21-30. 
[37] P.-L. Lions. Mathematical topics in fluid mechanics. Vol. 1, volume 3 of Oxford Lecture Series in Mathematics and its Applications. Oxford University Press, New York, 1996.

[38] J.-M. Rakotoson. Generalized solutions in a new type of sets for problems with measures as data. Differential Integral Equations, 6(1) (1993), 27-36.

[39] J.-M. Rakotoson. Uniqueness of renormalized solutions in a T-set for the $L^{1}$-data problem and the link between various formulations. Indiana Univ. Math. J., 43(2) (1994), 685-702.

[40] M. Sanchon and M. Urbano. Entropy solutions for the $p(x)$-Laplace equation. To appear in Trans. American Math. Soc.

[41] M. Troisi. Teoremi di inclusione per spazi di sobolev non isotropi. Ricerche. Mat., 18 (1969), 3-24.

[42] A. Youssfi, A. Benkirane and M. El Moumni. Bounded solutions of unilateral problems for strongly nonlinear equations in Orlicz spaces. Electronic Journal of Qualitative Theory of Differential Equations (EJQTDE), Number $21,(2013)$ pp: 1-25.

[43] A. Youssfi, A. Benkirane and M. El Moumni. Existence result for strongly nonlinear elliptic unilateral problems with $L^{1}$-data. Complex Variables and Elliptic Equations, Volume 59, Issue 4 ,(2014) pp: 447-461 . 